\title{
S4: Small State and Small Stretch Compact Routing Protocol for Large Static Wireless Networks
}

\author{
Yun Mao, Feng Wang, Lili Qiu, Senior Member, IEEE, Simon Lam, Fellow, IEEE, and Jonathan Smith, Fellow, IEEE
}

\begin{abstract}
Routing protocols for large wireless networks must address the challenges of reliable packet delivery at increasingly large scales and with highly limited resources. Attempts to reduce routing state can result in undesirable worst-case routing performance, as measured by stretch, which is the ratio of the hop count of the selected path to that of the optimal path. We present a new routing protocol, Small State and Small Stretch $(\mathrm{S4})$, which jointly minimizes the state and stretch. $\mathrm{S} 4$ uses a combination of beacon distance-vector-based global routing state and scoped distance-vector-based local routing state to achieve a worst-case stretch of 3 using $O(\sqrt{N})$ routing state per node in an $N$-node network. Its average routing stretch is close to 1 . S4 further incorporates local failure recovery to achieve resilience to dynamic topology changes. We use multiple simulation environments to assess performance claims at scale and use experiments in a 42-node wireless sensor network testbed to evaluate performance under realistic RF and failure dynamics. The results show that $\mathrm{S} 4$ achieves scalability, efficiency, and resilience in a wide range of scenarios.
\end{abstract}

Index Terms-Compact routing, resilience, routing, scalability, simulation, testbed experiments, TOSSIM.

\section{INTRODUCTION}

$\mathbf{R}$ OUTING finds paths in a network along which to send data and is one of the most basic network functions. The effectiveness of routing protocols directly affects network scalability, efficiency, and reliability. With continuing growth in the sizes of wireless network, routing protocols must simultaneously achieve the following design goals.

- Small routing state: Using small amounts of routing state is essential to achieving network scalability. Many wireless devices are resource-constrained. For example, mica2 sensor motes have only $4 \mathrm{kB}$ RAM. Limiting routing

Manuscript received March 29, 2007; revised May 22, 2009; approved by IEEE/ACM TRANSACTIONS ON NETWORKING Editor D. Towsley. First published April 19, 2010; current version published June 16, 2010. This work was sponsored in part by National Science Foundation CNS-0546755, CNS-0916106, ANI-0319168, CNS-0434515, CNS-0721845, and CNS-0739347 and Olga and Alberico Pompa Professorship of Engineering and Applied Science at the University of Pennsylvania. An earlier version of this paper appeared in the Proceedings of the 2007 USENIX Symposium on Networked Systems Design and Implementation (NSDI).

Y. Mao was with the University of Texas at Austin, Austin, TX 78712 USA. He is now with AT\&T Research, Florham Park, NJ 07974 USA. (e-mail: maoy@ research.att.com).

F. Wang was with the University of Texas at Austin, Austin, TX 78712 USA. He is now with Meraki, San Francisco, CA 94103 USA. (e-mail: wangf@meraki.com).

L. Qiu and S. Lam are with the University of Texas at Austin, Austin, TX 78712 USA (e-mail: lili@cs.utexas.edu; lam@cs.utexas.edu).

J. Smith is with the University of Pennsylvania, Philadelphia, PA 19104 USA (e-mail: jms@ cis.upenn.edu).

Color versions of one or more of the figures in this paper are available online at http://ieeexplore.ieee.org.

Digital Object Identifier 10.1109/TNET.2010.2046645 state is necessary for such devices to form large networks. Moreover, limiting routing state also helps to reduce control traffic used in route setup and maintenance since the amount of routing state and control traffic is often correlated.

- Small routing stretch: Routing stretch is defined as the ratio between the cost of selected route and the cost of optimal route. Small routing stretch means that the selected route is efficient compared to the optimal route. It is a key quantitative measure of route quality and affects global resource consumption, delay, and reliability.

- Resilience: Wireless networks often experience frequent topology changes arising from battery outage, node failures, and environmental changes. Routing protocols should find efficient routes even in the presence of such changes.

Existing routing protocols either achieve small worst-case routing stretches with large routing state (e.g., shortest path routing) or achieve small routing state at the cost of large worst-case routing stretches (e.g., geographic routing and hierarchical routing). In this paper, we present the design and implementation of Small State and Small Stretch (S4), a new addition to the routing protocol design space. $\mathrm{S} 4$ achieves a desirable balance among these characteristics and is well suited to the large-scale wireless network setting (e.g., sensor networks).

We make the following contributions.

1) S4 is the first routing protocol that achieves a worst-case routing stretch of 3 in large wireless networks. Its average routing stretch is close to 1 .

2) S4's distance-guided local failure recovery scheme significantly enhances network resilience and is portable to other settings.

3) S4's scalability, effectiveness of resource use, and resilience are validated using multiple simulation environments and a 42-node sensor network testbed.

The rest of the paper is organized as follows. Section II discusses prior work. The S4 routing protocol is introduced in Section III. S4 performance in an ideal wireless environment (no wireless medium losses or collisions) is studied using high-level simulation in Section IV. Section V presents further evaluation results using a packet-level simulator (TOSSIM) to study S4 performance in more realistic large-scale wireless networks with a wireless medium and collisions. Section VI describes a final S4 evaluation performed on an experimental testbed, and Section VII summarizes and concludes the paper.

\section{RELATED WORK}

Routing is a well-studied problem, but large-scale wireless networks have introduced new challenges. Shortest path routing protocols (e.g., DSR [11], AODV [23], DSDV [22]) can find good routes, but are limited in scale by both control traffic 
and the amount of state required at each node. Consequently, routing in large-scale wireless networks has focused on minimizing storage and exchange of routing state and can be divided into geographic routing and hierarchical routing approaches.

In geographic routing, each node is assigned a coordinate reflecting its position in the network. Upon receiving a packet, a node selects a next-hop closer to the destination in the coordinate space. Some geographic routing protocols use geographic locations as node coordinates, while others use virtual coordinates based on network proximity. As connectivity in the coordinate spaces is not complete, these schemes must address getting "stuck" in a local minimum, where no neighbor is closer to the destination than the current node. Some proposals such as GFG [2], GPSR [12], GOAFR + [15], GPVFR [18] and variants use face traversal schemes that route packets on a planar graph derived from the original connectivity graph. Their delivery guarantees [6] depend on the assumption that the planarization algorithms (e.g., GG [7] and RNG [28]) can successfully planarize any network graph. These planarization algorithms typically assume a unit disk or quasi-unit disk model. However, these models can be inadequate for real wireless environments due to obstacles and multipath fading. Kim et al. [14] have shown that model failures in real radio environments can cause routing pathologies and persistent routing failures. CLDP [13] addresses the imperfect RF propagation problem using a right-hand probing rule to detect link-crossings and remove them to replanarize the graph. GDSTR [17] provides delivery guarantee without requiring planarization by avoiding routing across the face of planar graphs and instead routing packets through a spanning tree.

The geographic coordinate-based routing schemes have at least three difficulties for wireless networks. First, accurate geolocation either requires careful static setting or access to GPS, with consequences for cost and need for line-of-sight to satellites. Second, geographic distances may lack predictive value for network performance (e.g., loss rate). This may result in paths with poor performance. Third, even with GPS and ideal radios, the best routing stretch for geographic routing is $O\left(c^{2}\right)$ in GOAFR+ [15] and ARF [16], where $c$ is the length of the optimal path, and example topologies exist where this bound is tight [16].

Virtual coordinates reflecting underlying network connectivity address the first two difficulties, but still face the challenge of "dead ends," for which a recovery scheme is required. In addition, the overhead of computing and storing virtual coordinates is not negligible. For example, NoGeo [25] uses $O(\sqrt{N})$ perimeter nodes to flood the $N$-node network so that every node can learn its distances to all the perimeter nodes. Each node determines its virtual coordinate based on the distances to the perimeter nodes. However, perimeter nodes need to store $O(N)$ pair-wise distance among them, which is not scalable in large wireless networks with limited memory space per node. GEM [21] achieves greater scalability by using triangulation from a root node and two other reference nodes. However, the routing stretch is larger than that of typical geographic routing algorithms, and there is the additional cost of recomputing routing labels resulting from network failures. Fonseca $e t$ al. [5] have proposed Beacon Vector Routing (BVR), which selects a few beacon nodes and uses flooding to construct spanning trees from the beacons to all other nodes. A node's coordinate is a vector of distances from the node to all beacons, and each node maintains the coordinates of its neighbors. BVR defines a distance metric over these beacon vectors, and a node routes packets to the one that minimizes the distance. When greedy routing stalls, it forwards the packet toward the beacon closest to the destination. If the beacon still fails to make greedy progress, scoped flooding is used. None of the virtual coordinate-based routing algorithms provide worst-case routing stretch guarantees.

Hierarchical routing is an alternative approach to achieving scalability. Example protocols in this category include landmark routing [29], LANMAR [8], ZRP [9], and Safari [24]. Hierarchical routing protocols provide no guarantee on the routing stretch due to boundary effects: Two nodes that are physically close may belong to different clusters or zones, and hence the route between them has to go through cluster heads, which can be arbitrarily longer than their shortest path.

Caesar et al. develop VRR [3], a scheme for layer-3 any-to-any routing based on distributed hash tables. To route to its successors on the virtual ring, a node sets up and maintains forwarding entries to its successors and predecessors along multihop physical paths. As a result, a node has both routing table entries toward its neighbors in the ring and also entries for the nodes on the paths in between. VRR greedily forwards a packet toward the node in the routing table with the closest ID to the destination ID. The routing state per node is roughly $O(\sqrt{N})$. Unlike S4, VRR does not provide worst-case routing stretch guarantee.

Theoretical work [4], [27] on achieving scalable and efficient routing has developed compact routing algorithms that provide a worst-case routing stretch of 3 while using at most $O(\sqrt{N \log N})$ state in an $N$-node network. This worst-case routing stretch is provably optimal when each node uses less than linear routing state [4], [27]. While compact routing seems to be a promising direction for large-scale networks, it cannot be directly translated into a routing protocol in a distributed network. In particular, the proposed algorithms do not specify how each node should build and maintain routing state for local clusters and for beacon nodes. Moreover, the algorithm in [27] requires choosing beacon nodes offline, considers only initial route construction, and cannot cope with topology changes, which precludes realization in our network setting. The implications of compact routing for average routing stretch also remain unclear.

\section{S4 RouTing PROTOCOL}

S4 uses the theoretical ideas of the compact routing algorithm [27] as a basis, refined by the addition of new techniques needed to obtain a practical routing protocol for large-scale wireless networks. We first describe the basic routing algorithm and identify challenges for routing protocol design, and then present the S4 routing protocol. Throughout this paper, our metric for the cost of a route is the number of links traversed (i.e., hop count).

\section{A. Basic Routing Algorithm}

In $\mathrm{S} 4$, a random set of nodes, $L$, is chosen as beacons. For a node $d$, let $L(d)$ denote the beacon closest to node $d$, and let 


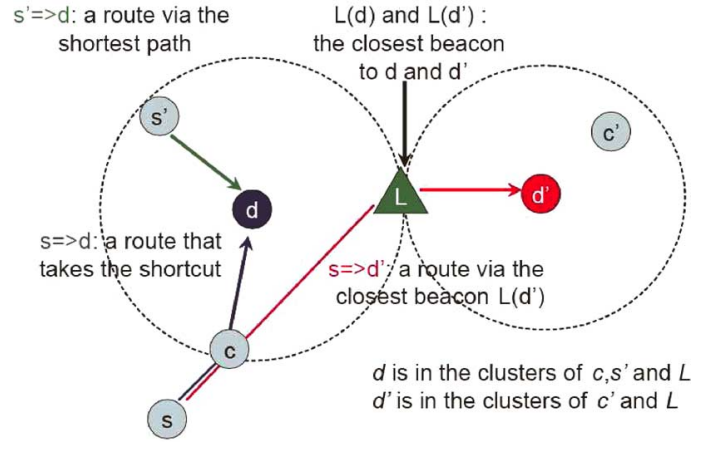

Fig. 1. S4 routing examples. Every node within the circle of $d$ has $d$ in its local cluster. The route $s^{\prime} \rightarrow d$ is the shortest path. The route $s \rightarrow d$ takes a shortcut at $c$ before reaching $L(d)$. The route $s \rightarrow d^{\prime}$ is through $L\left(d^{\prime}\right)$ without shortcut.

$\delta(s, d)$ denote the shortest path distance from $s$ to $d$. Each node $s$ constructs the following local cluster, denoted as $C_{k}(s)$ :

$$
C_{k}(s)=\{c \in V \mid \delta(c, s) \leq k * \delta(c, L(c))\}, \quad k \geq 1 .
$$

where $V$ is the set of all nodes in the network. A local cluster of node $s$ consists of all nodes whose distances to $s$ are within $k$ times their distances to their closest beacons. Each node $s$ then maintains a routing table for all beacon nodes and nodes in its own cluster $C_{k}(s)$.

As shown in Fig. 1, when routing from node $s$ to node $d$, if $d \in C_{k}(s)$, we can directly use the shortest path to route from $s$ to $d$. Otherwise, $s$ first takes the shortest path toward $L(d)$, and then uses the shortest path to route toward $d$. In the second case, the route does not have to always reach $L(d)$ before routing to $d$. Whenever data reaches a node $c$ whose cluster contains $d, c$ can directly route to $d$ using the shortest path from $c$ to $d$. According to the triangle inequality, the "shortcut" strictly improves routing stretch. We give the following theorem as an extension to the proof in [4] and [27], in which a special case $k=1$ is proved.

Theorem 1: Let $C_{k}(s)=\{c \in V \mid \delta(c, s)<k * \delta(c, L(c))\}$, where $k \geq 1$. If each node $s$ maintains next-hop for the shortest path to every beacon and every node in $C_{k}(s)$, the worst-case routing stretch is $1+\frac{2}{k}$.

Proof: When $d \in C_{k}(s)$, routing stretch is 1 since we know the shortest path from $s$ to $d$. When $d \notin C_{k}(s)$, let $r(s, d)$ denote the cost of selected route from $s$ to $d$.

$$
\begin{aligned}
r(s, d) & \leq \delta(s, L(d))+\delta(L(d), d) \\
& \leq \delta(s, d)+2 \delta(L(d), d) \\
& \leq \delta(s, d)+\frac{2}{k} \delta(s, d) \\
& =\left(1+\frac{2}{k}\right) \delta(s, d)
\end{aligned}
$$

The first inequality is due to possible shortcut before reaching $L(d)$. As shown in Fig. 1, the shortcut $c \rightarrow d$ is less than $c \rightarrow$ $L(d) \rightarrow d$ according to triangle inequality. Hence, $s \rightarrow c \rightarrow d$ is less than $s \rightarrow L(d) \rightarrow d$. Equality holds when there is no shortcut. The second inequality is due to triangle inequality and symmetry: The shortest path $s \rightarrow L(d)$ should cost no more than $s \rightarrow d \rightarrow L(d)$. Finally, the third inequality is based on the definition of cluster $C_{k}(s)$ and the fact that $d \notin C_{k}(s)$. This completes the proof.

As a special case, when $k=1$, a local cluster of node $s$ consists of all nodes whose distances to $s$ are closer than their distances to their closest beacons. This special case is called compact routing [4], [27]. It is particularly interesting since it has low worst-case storage cost of $O(\sqrt{N \log N})$ and provides a worst-case routing stretch of 3 . In the remaining paper we consider $k=1$ since it gives small routing state.

Practical concerns dictate three changes to the TZ compact routing scheme [27] to achieve S4. First, the boundary conditions of the cluster definitions are slightly different. In S4, $C(s)=\{c \in V \mid \delta(c, s) \leq \delta(c, L(c))\}$, but in the TZ scheme, $C(s)=\{c \in V \mid \delta(c, s)<\delta(c, L(c))\}$. That is, node $c$ is in the cluster of $s$ in S4 but not in the TZ scheme, if $\delta(c, s)=$ $\delta(c, L(c))$. This change does not affect the worst-case routing stretch, and reduces average-case routing stretch at the cost of increasing routing state.

Second, to route toward node $d$, only $L(d)$ should be carried in the packet header as the location information in S4. In comparison, the TZ scheme requires a label $(d)=(L(d), \operatorname{port}(L(d), d))$ for each packet, where $\operatorname{port}(L(d), d)$ is the next-hop at $L(d)$ toward $d$. Only with the label carried in the packet header, a beacon node can forward a packet toward $d$ using next-hop port $(L(d), d)$. It is necessary in the TZ scheme because the beacon nodes do not store routing state. However, in S4, as a result of the boundary condition change, each beacon node $L$ stores routing state to all the nodes that have $L$ as its closest beacon node. Given that the total storage cost of the additional field port $(L(d), d)$ in the labels is the same as the total number of routing entries at beacon nodes in S4 (i.e., both are $N$ ), we favor storing routing state at beacon nodes since it reduces packet header length and the frequency of updating labels. The frequency of label updates is reduced because labels are updated only when $L(d)$ changes but not when $\operatorname{port}(L(d), d)$ changes.

Finally, the TZ scheme proposes a centralized beacon node selection algorithm to meet expected worst-case storage bound $O(\sqrt{N \log N})$ in an $N$-node network. Since practicality is our main design goal, in $\mathrm{S} 4$ we randomly select beacon nodes in a distributed fashion. It is proved that when $O(\sqrt{N})$ nodes are randomly selected as beacon nodes, the average storage cost on each node is still $O(\sqrt{N})$ [26]. As our evaluation results show, the storage cost is still low even for the worst cases. Note that the worst-case routing stretch of 3 still holds under random beacon node selection.

\section{B. Design Challenges}

Designing a routing protocol to realize the algorithm proposed in Section III-A poses the following challenges.

First is how to construct and maintain routing state for a local cluster. Frequent topology changes in wireless networks make it necessary to support incremental routing updates. Unlike traditional hierarchical routing, each node has its own cluster in compact routing. Therefore, naive routing maintenance could incur significant overhead.

Second is how to construct and maintain routing state for beacon nodes. Knowledge of next-hops and shortest path distances to beacon nodes is important to the performance of S4. 
When beacon packets are lost, the routing state could be inaccurate, which could substantially degrade the performance.

Third is how to provide resilience against node/link failures and environmental changes. Maintaining up-to-date routing state could be expensive, especially in a large network. Moreover, routing changes take time to propagate. During the transient period (e.g., the period from the time when failure occurs to the time when the routing tables at all nodes are updated to account for the failure), many packets could be lost without a failure recovery scheme.

To address the above challenges, $\mathrm{S} 4$ consists of the following three major components: 1) scoped distance vector for building and maintaining routing state to nodes within a cluster; 2) resilient beacon distance vector for efficient routing toward beacon nodes and facilitating intercluster routing; and 3) distance-guided local failure recovery for providing high-quality routes even under dynamic topology changes. We will describe these three components in turn.

\section{Intracluster Routing: Scoped Distance Vector (SDV)}

In $\mathrm{S} 4$, node $s$ uses the shortest paths to route toward nodes in the cluster of $s$. Unlike the traditional hierarchical routing, in S4 each node $s$ has its own cluster, which consists of nodes close to node $s$. This clustering is essential for providing a routing stretch guarantee since it avoids boundary effects. In comparison, hierarchical routing cannot provide routing stretch guarantee due to boundary effects, where two nearby nodes belong to different clusters and the hierarchical route between them could be much longer than their direct shortest path.

A natural approach to building a local routing table is to use scoped flooding. That is, each node $d$ floods the network up to $\delta(d, L(d))$ hops away from $d$, where $\delta(d, L(d))$ is the distance between $d$ and its closest beacon $L(d)$. Scoped flooding works fine when the network is initialized or when there are new nodes joining the network. However, it is costly to send frequent scoped flooding to reflect constant topology changes, which often arises in wireless networks due to battery outage, node failures, and environmental changes.

Scoped distance vector: To provide cheap incremental routing updates, we propose using scoped distance vector (SDV) for constructing routing tables for local clusters. SDV is attractive because it is fully distributed, asynchronous, and supports incremental routing updates. SDV is more efficient than scoped flooding, especially under small changes in a network topology because a node in SDV propagates routing update only when its distance vector changes, while in scoped flooding, a node propagates a flooded packet regardless of whether its distance and next-hop to a destination have changed.

In $\mathrm{S} 4$, each node $s$ stores a distance vector for each destination $d$ in its cluster as the following tuple:

$$
\langle d, \text { nexthop }(s, d), \delta(s, d), \operatorname{seqno}(d), \text { scope }(d), \text { updated }\rangle
$$

where $d$ and nexthop $(s, d)$ are both node IDs, seqno is the latest sequence number for destination $d$, $\operatorname{scope}(d)$ is the distance between $d$ and $d$ 's closest beacon, and updated is whether the distance vector has been updated since the last routing update.

A node $s$ exchanges its distance vectors with its neighbors either synchronously or asynchronously. Node $s$ initializes $\delta(s, c)=1$ for only $c \in$ neighbor $(s)$, and $\infty$ otherwise. Upon receiving a distance vector, a node $c$ uses the newly received distance vectors to update its routing state. Node $c$ further propagates the update for $s$ only when its current distance from $s$ is below scope $(s)$ and its distance vector to $s$ has changed.

Benefits of SDV: SDV supports incremental routing updates. This allows a wireless network to dynamically adapt to routing changes. Moreover, unlike traditional distance vector protocols, SDV does not suffer from the count-to-infinity problem ${ }^{1}$ because the scope is typically small (e.g., we evaluate a 1000-node network with 32 beacons, and its average scope is 3.35 and maximum scope is 13 . This implies routing loops can be detected within 13 hops).

\section{Intercluster Routing: Resilient Beacon Distance Vector $(R B D V)$}

To support routing across clusters, each node is required to know its distances to all beacons. This can be achieved by constructing a spanning tree rooted from each beacon nodes to every other node in the network. Flooding beacon packets reliably is important to the routing performance because loss of beacon packets may introduce errors in estimating the closest beacon and its distance and degrade the performance of S4. We develop a simple approach to enhance resilience of beacon packets.

Routing state construction and maintenance: To construct routing state for beacon nodes, every beacon periodically broadcasts beacon packets, which are flooded throughout the network. Every node then keeps track of the shortest hop count and next-hop toward each beacon.

Since beacon packets are broadcast and typical MAC protocols (e.g., CC1000 used in sensor motes) do not provide reliability for broadcast packets, it is essential to enhance the resilience of beacon packets at the network layer. Our idea is to have a sender retransmit the broadcast packet $P$ until $T$ fraction of neighbors have forwarded $P$ or until the maximum retry count Retry $_{\max }$ is reached. $T$ and Retry max $_{\max }$ provide a tradeoff between overhead and reliability. In our evaluation, we use Retry $_{\max }=3, T=100 \%$ for beacon nodes, and $T=1 / 3$ for nonbeacon nodes, which corresponds to the following condition in our implementation (to avoid floating point calculation): $3 *$ \# neighbors forwarded $\mathrm{P} \geq \#$ neighbors. $T=100 \%$ for a beacon node is used because all neighbors of the beacon nodes should forward the beacon packet. In comparison, for a nonbeacon node $c$, only a subset of $c$ 's neighbors are farther away from the beacon than $c$ and need to forward the beacon packet received from $c$. Therefore, we use a smaller $T$ for nonbeacon nodes.

\section{E. Distance-Guided Local Failure Recovery (DLF)}

Wireless networks are subject to temporary or permanent node/link failures due to obstruction, signal fading, energy depletion, or physical damage [5], [1], [20]. To provide high routing success rate and low routing stretch even in the presence of such failures, we develop a simple and effective local failure recovery based on distance vectors.

Overview: To achieve high resilience, $\mathrm{S} 4$ provides failure recovery at the network layer in additional to MAC-layer

\footnotetext{
${ }^{1}$ The count-to-infinity problem is that when a link fails, it may take a long time (on the order of network diameter) before the protocol detects the failure. During the interim, routing loops may exist.
} 


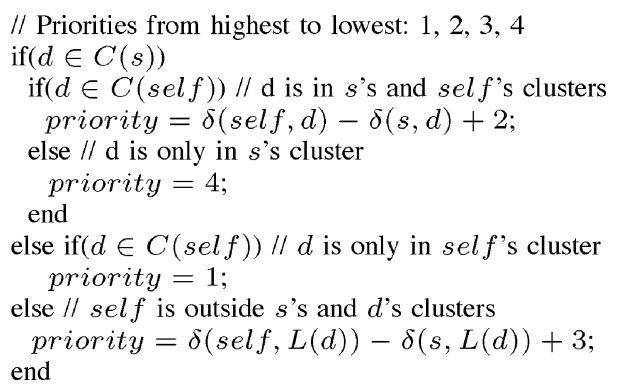

Fig. 2. Computing priority using scoped distance vectors and beacon distance vectors.

retransmission. Specifically, a node $s$ retransmits a packet at the network layer when it does not receive an ACK even with MAC-layer retransmission. When $R$ retransmissions at the network layer fail, $s$ broadcasts a failure recovery request, which contains: 1) the next-hop $s$ used; 2) whether destination $d$ is included in $s$ 's local cluster; and 3) the distance to $d$ if $s$ 's cluster includes $d$, or the distance to $d$ 's beacon otherwise. Upon hearing the failure requests, $s$ 's neighbors attempt to recover the packet locally. Our goal is to select the neighbor that is the closest to the destination as $s$ 's new next-hop; meanwhile, the selection process should be cheap and easily distributed.

S4 uses distance-guided local failure recovery to prioritize neighbors' responses based on their scoped distance vectors. Each node uses its priority to determine the time it needs to wait before sending failure recovery response. We further exploit broadcast nature of wireless medium to avoid implosion of recovery responses. Note that if no response is received, $s$ can retransmit the failure recovery request up to a threshold. Our evaluation uses a threshold of 0 (i.e., $s$ does not retransmit), and we already see significant performance improvement. With more retries, the improvement would be even higher.

Distance-guided local failure recovery: Our goal is to prioritize neighbors based on their distances to the destination so that the nodes closest to the destination can take over the forwarding. The problem is nontrivial because the distance to the destination is not always available. When the destination is outside the local cluster, a neighbor only knows the distance to the destination's closest beacon, but not the distance from that beacon to the destination.

To address the issues, each node computes its priority using the algorithm in Fig. 2. It involves two main scenarios. In the first scenario, $s$ 's local cluster contains the destination $d$. This information is available in $s$ 's failure recovery request. Then, $s$ 's neighbor is assigned one of the four priorities using the following rules. The neighbors that have $d$ in their clusters are assigned the top three priorities since they can directly route toward the destination using the shortest path. In this case, each neighbor knows its distance to the destination and assigns itself a priority based on the difference between $\delta$ (self, $d)$ and $\delta(s, d)$. Neighbors whose local clusters do not contain the destination are assigned the fourth priority, which is the lowest.

In the second case, when $s$ 's cluster does not contain the destination $d$, only the neighbors that have $d$ in their clusters are assigned the highest priority since they can directly route toward the destination. The other nodes are assigned priorities by comparing their distances to the beacon with $\delta(s, L(d))$.
A sender $s$ selects the neighbor from which it receives the response first as the new next-hop. By assigning each neighbor $i$ with a timer priority $(i) \times m+$ rand, a higher priority node sends the response earlier and is thus favored as the new next-hop node. To avoid collisions, we add a small random timer rand to the priority-based timer so that different nodes are likely to respond at different times even when assigned the same priority. To avoid response implosion, upon hearing a failure response to $s$ from someone else, the current node cancels its own pending recovery response if any. Our evaluation uses $m=50 \mathrm{~ms}$, and rand ranges from 0 to $49 \mathrm{~ms}$.

Node failures versus link failures: The above scheme works well for link failures. When a node fails, all the links to and from the failed nodes are down. Therefore, we need to avoid using nodes that use the failed nodes as next-hop. This can be done by letting the sender specify the failed node. Only the nodes that use different next-hop from the failed node will attempt to recover. To identify a failed node, every node periodically broadcasts a hello message once every $30 \mathrm{~s}$, and a node considers its neighbor as failed if it does not receive any hello messages from that neighbor for the last five hello intervals.

\section{F. Other Design Issues}

Location directory: So far, we assume that the source knows which beacon node is closest to the destination. In practice, such information may not be directly available. In such a situation, the source can apply the location directory scheme described in BVR [5] to look up such information. More specifically, beacon nodes are responsible for storing the mapping between nonbeacon nodes and their closest beacons. The closest beacon information for node $i$ is stored at $H(i)$, where $H$ is a consistent hash function that maps nodeid to beaconid. The source contacts the beacon node whose ID is $H$ (dest) to obtain the closest beacon to dest. The storage cost of location directory is much smaller in S4 than that in BVR (as shown in Section IV) because the source in S4 only needs to know the closest beacon to its destination, while the source in BVR needs to know the distance between its destination and all beacon nodes. Moreover, in S4 when destination $d$ is in $s$ 's cluster, no location lookup is required since $s$ knows the shortest path to $d$, whereas BVR as well as other geographic routing schemes always require location lookup on a new destination. Such property is especially beneficial when traffic exhibits locality (i.e., nodes close to each other are more likely to communicate).

Beacon maintenance: When a beacon fails, S4 applies distance-guided local failure recovery to temporarily route around the failure. If the failure persists, we can apply the beacon maintenance protocol proposed in [5] to select a new beacon. Beacon maintenance is not the focus of this paper. Instead, we focus on the routing performance during the transient period after failures occur.

Link quality: Link quality significantly affects routing performance. We define link quality as the delivery rate of packet on the link in a given direction. In S4, each node continuously monitors its links to/from its neighbors. We adopt a passive link estimator layer developed in [5] and [30] for estimating link quality. When a node receives a beacon packet or SDV update, it first checks if both the forward and reverse link qualities of 
the sender are above a threshold ( $30 \%$ is used in our current implementation). Only those updates from a sender with good link quality in both directions will be accepted.

\section{Simulation}

In this section, we evaluate the efficiency and scalability of S4 by simulation. We compare S4 with BVR [5] because BVR is one of the latest scalable routing protocols and also among the few that have been implemented in real sensor networks. We use BVR with scoped flooding since it provides delivery guarantee and offers a fair baseline comparison. We use three evaluation methodologies: 1) MATLAB simulation based on the unit disk graph radio model (presented in this section); 2) TOSSIM simulation, a packet-level simulator with more detailed wireless model (presented in Section V); and 3) testbed evaluation (presented in Section VI). Our MATLAB simulation results can be directly compared to many previous works on geographic routing in which the unit disk model is used. TOSSIM simulations allow us to study the performance in more realistic large-scale wireless networks. Having both levels of simulations also reveals how underlying wireless models may affect the routing performance. For BVR, we validate our MATLAB implementation of BVR by comparing with the original BVR simulation code, and we directly use the original BVR implementation in TinyOS for TOSSIM evaluation.

\section{A. Simulation Methodology}

To study the protocols in an ideal wireless environment, $N$ nodes are randomly placed in a square rectangular region of size $A^{2}$ in the simulator. The packet delivery rates among nodes are derived from the unit disk graph model. That is, each node has a fixed communication range $R$. A node can communicate with all the nodes inside $R$, but cannot communicate with any node outside $R$. It is also assumed that there is no packet loss, collision, or network congestion. In the following description, we let $N$ denote the number of nodes, $K$ denote the number of beacon nodes, $R$ denote communication range, and $A^{2}$ denote the size of the area.

We use the following performance metrics to quantify the efficiency and robustness of S4:

- Routing stretch: the ratio of the route length using the selected routing protocol to that using the optimal shortest path routing protocol.

- Transmission stretch: the ratio of the total number of packets transmitted using the selected routing protocol to that using the optimal shortest path routing protocol. It may differ from routing stretch because a single hop may sometimes require multiple transmissions (e.g., scoped flooding).

- Routing state: the amount of state required to maintain at each node.

- Control traffic: the amount of traffic transmitted for setting up the routing state and location directory.

Unless specified otherwise, our default simulation scenario uses a 3200-node network with nodes uniformly distributed in an area of $25 \times 25$ square units. The communication range $R$ is 1 unit. On average, each node has 15.4 immediate neighbors. Beacon nodes are randomly selected. In BVR, all or a subset of

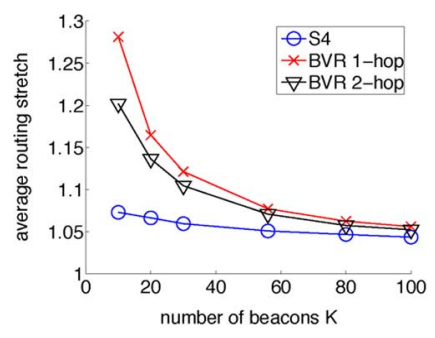

(a)

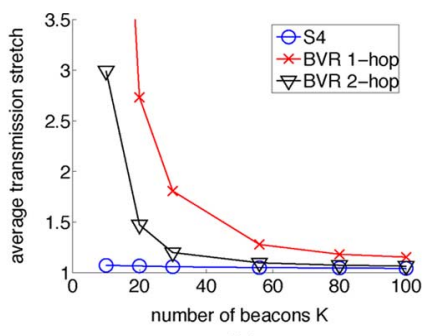

(b)
Fig. 3. S4 has routing and transmission stretches close to 1, which is consistently smaller than those of BVR algorithms across all numbers of beacons. (a) Routing stretch. (b) Transmission stretch.

beacon nodes serve as routing beacons; a node's coordinate is defined as its distances to the routing beacons. The number of routing beacons $K_{R}$ is fixed to 10 for all simulations because it is reported to offer a good balance between routing performance and overhead [5]. For each configuration, we conduct 10 random runs and report the aggregate statistics.

\section{B. Simulation Results}

1) Varying the Number of Beacons $(K)$ : Routing and transmission stretches: First, we compare the routing and transmission stretches of $\mathrm{S} 4$ and two variants of BVR by varying the number of beacons $K$. BVR 1-hop refers to the default BVR algorithm. BVR 2-hop is an on-demand 2-hop neighbor acquisition. In this approach, when a node cannot use greedy forwarding to make progress, it fetches its 1-hop neighbors' neighbors to its routing table. BVR 2-hop reduces the routing failure rate of BVR 1-hop at the cost of higher routing state and control traffic.

Fig. 3(a) compares the routing stretches under S4, BVR 1-hop, and BVR 2-hop. The stretches are computed based on 32000 routes between randomly selected pairs of nodes. We observe that S4 has the lowest average routing stretch. A closer examination of the simulation results shows that the worst stretches in S4 are bounded by 3. This is consistent with the worst-case guarantee provided by $\mathrm{S} 4$. In comparison, the average routing stretches in BVR 1-hop and 2-hop are substantially higher especially for small $K$. Moreover, their worst-case routing stretches are even higher (e.g., the worst routing stretch of BVR 1-hop in the simulation is 6 for $K=56$, and much larger for smaller $K$ ).

Fig. 3(b) compares transmission stretch among the three routing protocols. The average transmission stretches of S4 are consistently below 1.1 under all values of $K$. However, both BVR 1-hop and BVR 2-hop have much higher stretches when $K$ is small. To achieve comparable transmission stretches to S4 (though still higher), the least numbers of beacons required is 56 for BVR 1-hop and 30 for BVR 2-hop. Such high transmission stretch in BVR is due to its scoped flooding, which is necessary for its guaranteed delivery.

Routing state: Fig. 4 compares routing state per node under the three routing protocols. The routing state in S4 includes route entries for beacon nodes and for nodes within local clusters, whereas the routing state in BVR is determined by the number of neighbors and the length of their beacon vectors 


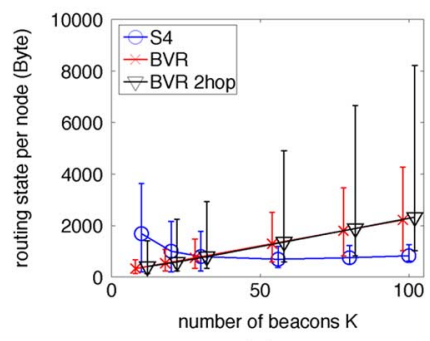

(a)

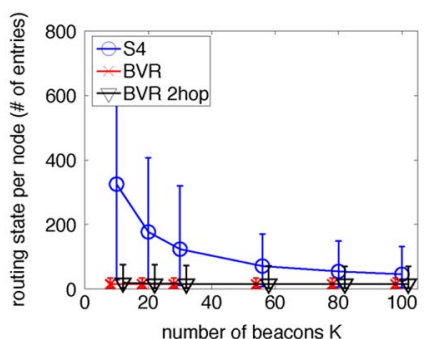

(b)

Fig. 4. Routing state comparison, where the errorbars show that when $K=$ $\sqrt{N}$, the routing state in $\mathrm{S} 4$ is half of routing state in BVR. (a) Number of bytes. (b) Number of routing table entries.

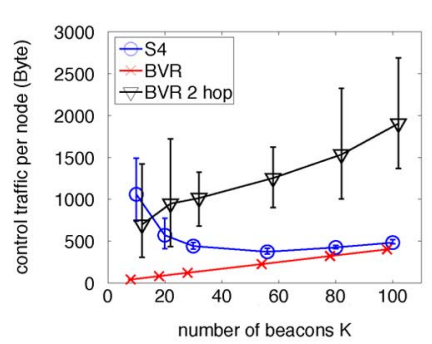

(a)

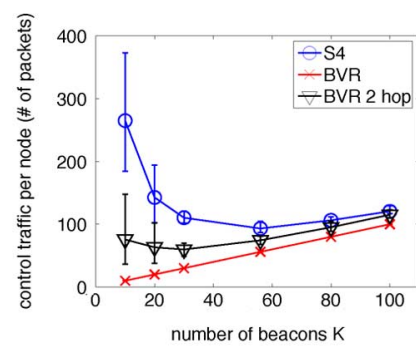

(b)

Fig. 5. Initial control traffic to set up routing state. The errorbars show minimum, mean, and maximum traffic across all nodes. The control traffic of S4 decreases gracefully as the number of beacons increases. When $K=\sqrt{N}$, the overhead of S4 is $65 \%$ higher than that of BVR 1-hop, but much less than BVR 2-hop. (a) Number of bytes. (b) Number of packets.

$K^{2}$. We make the following observations. First, in BVR the average routing table size proportionally increases with the number of beacons, while the number of entries remains close to the number of neighbors. In comparison, the routing state in $\mathrm{S} 4$ first decreases and then slightly increases with the number of beacon nodes. The routing state in $\mathrm{S} 4$ reaches minimum for $K \approx \sqrt{N}$ since it gives a good balance between global routing state (for beacon nodes) and local routing state (for nodes in the clusters). These trends also hold for maximum routing state in BVR and S4. Second, recall that to achieve a relatively small transmission stretch, 56 beacon nodes are required in BVR. In this case, the average and maximum routing state in BVR is twice or more than those of S4. Third, BVR 2-hop has significantly higher upper bound of routing state than BVR 1-hop due to the requirement of holding 2-hop neighbor information.

Control traffic: Fig. 5 shows initial control traffic for setting up routing state. The bandwidth overhead of BVR 1-hop increases linearly with the number of beacons because the main overhead is the beacon flooding messages. In BVR 2-hop, other than beacon flooding, the control traffic also includes the overhead of fetching 2-hop neighbor coordinates for the required nodes. We can see the overhead of on-demand 2-hop neighbor acquisition is significant, which is a big disadvantage of BVR 2-hop even though its routing stretch is lower than BVR 1-hop. In S4, control traffic includes beacon flooding and SDV. As $K$ increases, the size of the local cluster of each node decreases, so

\footnotetext{
${ }^{2}$ The size of a routing table entry in S4 is 5 bytes long in our implementation. The routing state of BVR is estimated based on the relevant data structures found in the BVR implementation code.
}

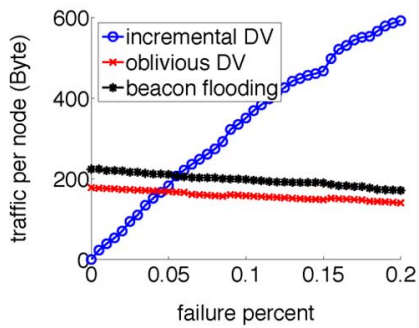

(a)

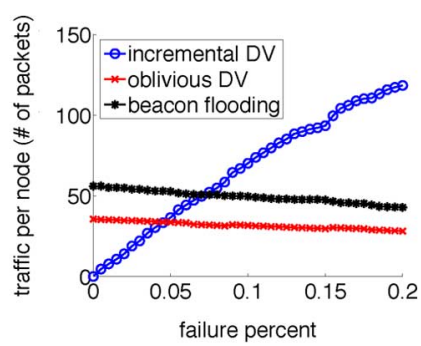

(b)
Fig. 6. Control traffic overhead of updating routing state due to topology changes. (a) Number of bytes. (b) Number of packets.

the number of scoped DV packets is reduced. When $K=56$, the overhead of S4 is $65 \%$ higher than that of BVR 1-hop. However, since SDV can be updated incrementally after the initial setup, its amortized overhead over the long run is reduced. In terms of the number of packets, S4 is less than twice of the BVR 1-hop when $K \geq \sqrt{N}$. Note that the number of packets in $\mathrm{S} 4$ can be reduced by grouping SDV packets. On the other hand, BVR demands large packet size when the number of beacons is large, and large packets could be forced to split in order to achieve high delivery rates under unreliable links.

To evaluate the overhead of incremental SDV in S4, we randomly select nonbeacon nodes to fail between two consecutive routing updates to create topology changes. There are two ways of updating the routing state after the initial round: either incrementally update based on the current routing state (incremental DV), or build new routing tables starting from scratch (regular DV). As shown in Fig. 6, when the number of node failures is small (e.g., within 5\%), incremental routing updates incur lower overhead. Since the typical number of node failures between consecutive routing updates is likely to be low, incremental routing updates are useful in real networks.

The control traffic to set up the routing table is not the only overhead. The source should be able to lookup the location information of the destination. Therefore, each node should store its location to a directory during the setup phase. We study such directory setup overhead by using the location directory scheme described in Section III-F: Each node $v$ periodically publishes its location to a beacon node $b_{v}$ by using a consistent hashing mechanism. $b_{v}$ then sends a confirmation back to $v$ if the publishing is successful. We simulate the initial directory setup overhead, in which every node publishes its location to the distributed directory. The results are shown in Fig. 7(a), and they include traffic to and from beacon nodes for publishing the locations. S4 has the following three advantages over the BVR. First, the size of location information in $\mathrm{S} 4$ is significantly smaller than that of BVR because, in BVR, a node's coordinate is proportional to the number of beacons, while in S4, a node's coordinate is its closest beacon ID. Second, the transmission stretch of BVR is higher than that of S4. Therefore, it incurs more traffic in routing a confirmation packet from the beacon node back to the node publishing its location. Third, it is more likely that a node changes its coordinates in BVR than it changes its closest beacon in S4. Therefore, S4 incurs a lower overhead in setting up and maintaining the location directory.

Fig. 7(b) shows the overall traffic overhead incurred in setting up both routing state and directory. We observe that compared 


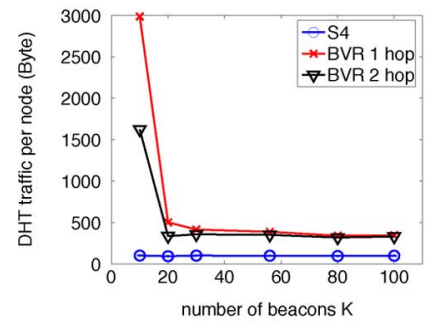

(a)

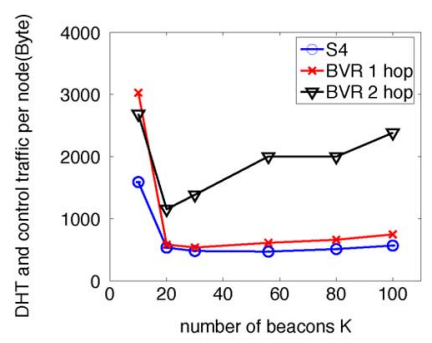

(b)
Fig. 7. Control traffic overhead comparison. (a) Location directory setup traffic. (b) Overall control traffic.

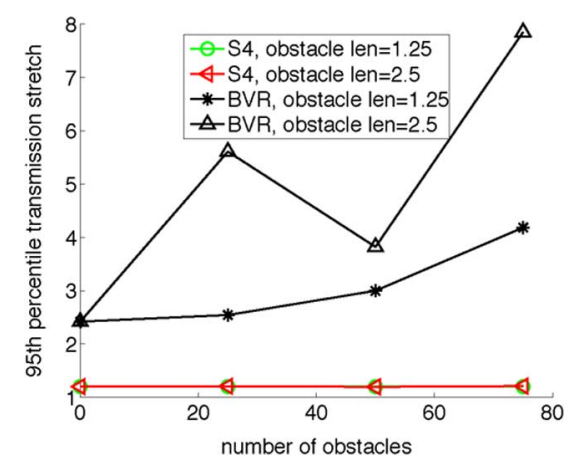

Fig. 8. Transmission stretch comparison between S4 and BVR in the presence of obstacles.

to both variants of BVR, S4 has smaller overall control traffic, including traffic in setting up both route and location directory.

Per-data packet header overhead: Aside from the control traffic, routing protocols also have overhead in the data packet headers. The overhead of S4 includes the closest beacon ID to the destination and its distance. For BVR, the overhead mainly depends on the number of routing beacons $K_{R}$. The packet header of BVR includes a $K_{R}$-long destination coordinate, which has at least $\left\lceil\log _{2}\left(\begin{array}{c}K \\ K_{R}\end{array}\right)\right\rceil$ bits indicating which $K_{R}$ nodes are chosen out of the total $K$ beacons as the routing beacons for the destination. For example, a rough estimation suggests that with $K=56$ and $K_{R}=10$, BVR requires 15 -byte packet headers, which is significant compared to the default packet payload size of 29 bytes in mica 2 motes, while S4 only takes 3 bytes in the packet header.

2) Under Obstacles: We now study the performance of S4 and BVR in the presence of obstacles using the same methodology as in [5]. The obstacles are modeled as horizontal or vertical walls, which completely block wireless signals. (They do not reflect wireless signals.) We vary the number and length of those randomly placed obstacles. We find that the median transmission stretches of S4 and BVR are 1.00 and 1.04, respectively. They are both insensitive to the obstacles. However, as shown in Fig. 8, the 95th percentile of the transmission stretches of S4 and BVR are quite different: S4 has a constant 95th percentile stretch around 1.2 regardless the existence of obstacles, while the transmission stretch of BVR increases with the number of the obstacles and the length of the obstacles. For example, when there are 75 obstacles with length 2.5 times of the transmission range, $12.9 \%$ of the links are blocked by them. As a result, the 95th percentile transmission stretch of BVR increases up to
7.9 due to the irregular topology, while the stretch of S4 stays around 1.2. This is because S4's worst-case routing stretch guarantee is independent of network topologies.

3) Summary: Our evaluation shows that $\mathrm{S} 4$ provides a worstcase routing stretch of 3 and an average routing stretch around 1.1-1.2 in all evaluation scenarios. When $K=\sqrt{N}$ (a favorable operating point for both S4 and BVR), S4 has significantly smaller routing state than BVR. While the initial route setup traffic in S4 is higher than that of BVR, due to its compact location representation, its total control traffic including location setup is still comparable to that of BVR. Furthermore, S4 can efficiently adapt to small topology changes using incremental routing update. Finally, BVR 1-hop is more scalable than BVR 2-hop due to its lower control traffic and routing state. So in the following evaluation, we only consider BVR 1-hop as a baseline comparison.

\section{TOSSIM EVALUATION}

We have implemented a prototype of S4 in nesC language for TinyOS [10]. The implementation can be directly used both in TOSSIM simulator [19] and on real sensor motes. In this section, we evaluate the performance of S4 using extensive TOSSIM packet-level simulations. By taking into account actual packet transmissions, collisions, and losses, TOSSIM simulation results are more realistic.

Our evaluation considers a wide range of scenarios by varying the number of beacon nodes, network sizes, network densities, link loss rates, and traffic demands. More specifically, we consider two types of network densities: a high density with an average node degree of 16.6 and a low density with an average node degree of 7.6. We use both lossless links and lossy links that are generated by LossyBuilder in TOSSIM. Note that even when links are lossless, packets are still subject to collision losses. In addition, we examine two types of traffic: a single flow and five concurrent flows. The request rate is one flow per second for single-flow traffic and five flows per second for 5-flow traffic. The simulation lasts for $1000 \mathrm{~s}$. Therefore, the total number of routing requests is 1000 for single-flow traffic and 5000 for 5-flow traffic. We compare S4 to BVR, whose implementation is available from the public CVS repository of TinyOS.

\section{A. Routing Performance}

First, we compare S4 with BVR under stable network conditions. To reach stable network conditions, we let each node periodically broadcast RBDV and SDV packets every $10 \mathrm{~s}$. Data traffic is injected into the network only after route setup is finished. BVR uses scoped flooding after a packet falls back to the beacon closest to the destination and greedy forwarding still fails, whereas S4 uses the distance-guided failure recovery scheme to recover failures. To make a fair comparison, in both BVR and S4, beacon nodes periodically broadcast and build spanning trees, and RBDV is turned off in S4.

1) Varying the Number of Beacons: We vary the number of beacon nodes from 16 to 40 while fixing the total number of nodes to 1000 .

Routing success rate: We study four configurations: a single flow with lossless links, a single flow with lossy links, five flows with lossless links, and five flows with lossy links. In the interest 


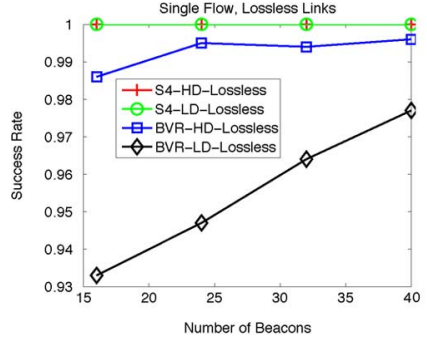

(a)

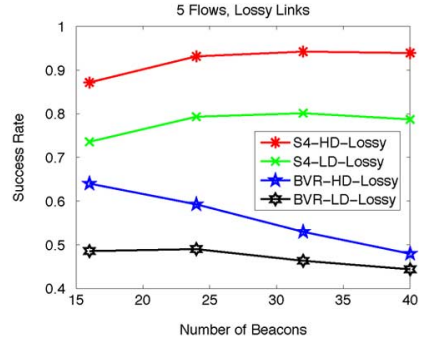

(b)
Fig. 9. Compare routing success under different numbers of beacons, network densities, and traffic patterns. (a) Lossless links with one flow. (b) Lossy links with five flows.

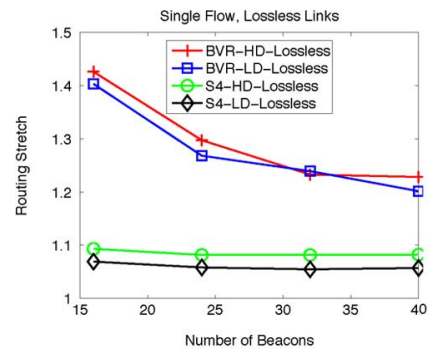

(a)

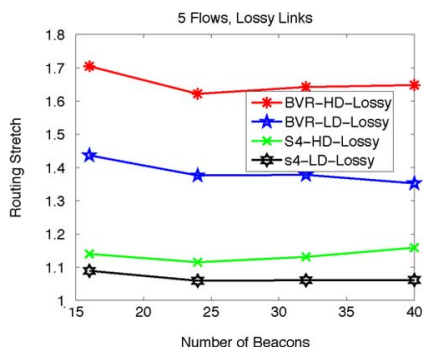

(b)
Fig. 10. Compare routing stretch under different numbers of beacons, network densities, and traffic patterns. (a) Lossless links with one flow. (b) Lossy links with five flows.

of space, Fig. 9 only shows the results of the first and last configurations. "HD" and "LD" curves represent results under high and low network densities, respectively.

We make the following observations. First, under lossless links with ove flow, S4 always achieves $100 \%$ success rate. In comparison, BVR achieves close to $100 \%$ success only in high-density networks, but its success rate reduces to $93 \%$ under low network density with 16 beacons. Why does BVR not provide delivery guarantee even under perfect channel condition? The reason is that scoped flooding is invoked after a packet is stuck at the fallback beacon, and scoped flooding could cause packet collisions and reduce packet delivery rate.

Second, under lossy links with five flows, packet losses are common, and the performance of both S4 and BVR degrades. Nevertheless, S4 still achieves around $95 \%$ routing success rate in high-density networks, while success rate of BVR drops dramatically. The large drop in BVR is because its scoped flooding uses broadcast packets, which have no reliability support from MAC layer; in comparison, data packets are transmitted in unicast under $\mathrm{S} 4$ and benefit from link layer retransmissions. Third, the success rate is lowest under low-density networks with lossy links and five flows. Even in this case, S4 achieves 70\%-80\% success rate, while the success rate of BVR is reduced to below $50 \%$.

Routing stretch: Fig. 10 compares the average routing stretch of S4 and BVR. The average routing stretch is computed only for the packets that have been successfully delivered. Although the worst stretch of S4 is 3 , its average stretch is only around 1.1-1.2 in all cases. In comparison, BVR has significantly larger routing stretch: Its average routing stretch

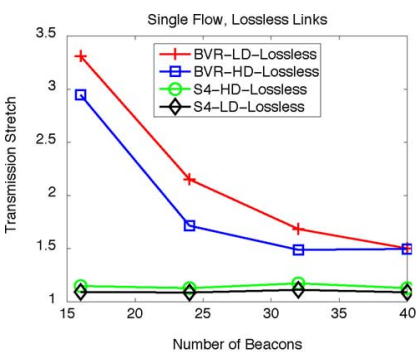

(a)

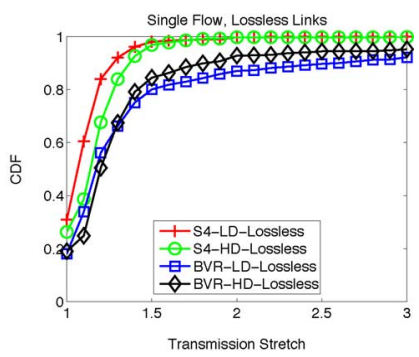

(b)
Fig. 11. Transmission stretch comparison. (a) Average transmission stretch. (b) $\mathrm{CDF}$ of transmission stretch.

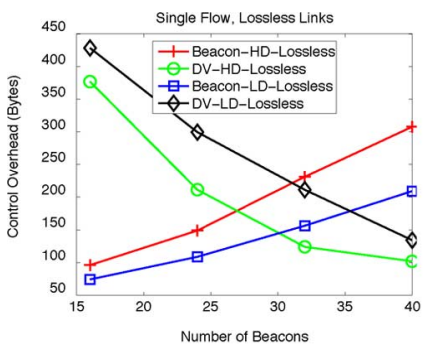

(a)

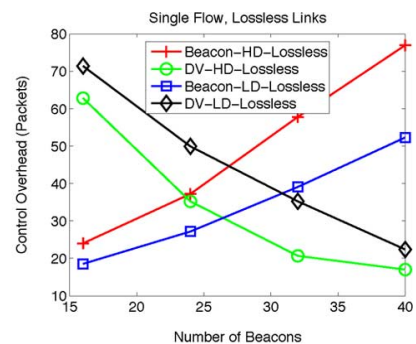

(b)
Fig. 12. Control traffic overhead under different numbers of beacons and network densities. (a) Control traffic in bytes. (b) Control traffic in packets.

is $1.2-1.4$ for one flow, and $1.4-1.7$ for five flows. Moreover, its worst routing stretch (not shown) is 8 .

Transmission stretch: As shown in Fig. 11(a), the transmission stretch of S4 is close to its routing stretch, while the transmission stretch of BVR is much larger than its routing stretch due to its scoped flooding, which lets all nodes within the flooding scope perform transmission and significantly increases transmission stretch. Fig. 11(b) shows the cumulative distribution function (CDF) of transmission stretches under 32 beacon nodes. We observe that the worst-case transmission stretch in $\mathrm{S} 4$ is 3 , and most of the packets have transmission stretch very close to 1 .

Control traffic overhead: Compared to BVR, S4 introduces extra control traffic of SDV to construct routing tables for local clusters. To evaluate this overhead, we count the average control traffic (in bytes and number of packets) that each node generates under lossless links and a single flow. We separate the global beacon traffic and local SDV traffic. The results are shown in Fig. 12. Note that beacon traffic overhead is the same for both S4 and BVR.

We can see that when the number of beacons is small, the SDV traffic dominates since the cluster sizes are relatively large in such a case. As the number of beacons increases, the amount of SDV traffic decreases significantly. In particular, when there are 32 beacons $(\approx \sqrt{1000})$, the amount of SDV traffic is comparable to the amount of global beacon traffic. Moreover, if we include control traffic for setting up location directory, the total control traffic in S4 would be comparable to that of BVR, as shown in Fig. 7.

Routing state: We compare routing state of S4 and BVR as follows. For S4, the routing state consists of a beacon routing table and a local cluster table. For BVR, the routing state consists of a beacon routing table and a neighbor coordinate table. 


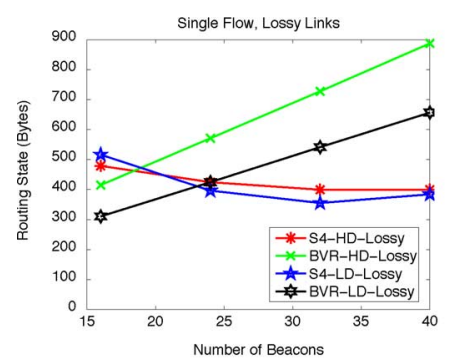

(a)

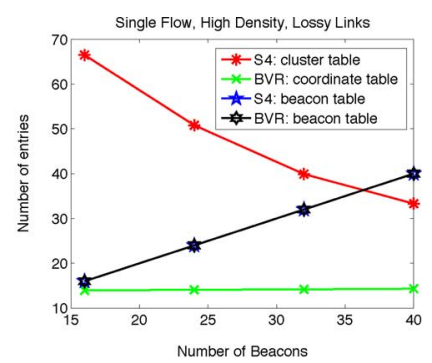

(b)
Fig. 13. Routing state comparison under different numbers of beacons and network densities with lossy links (single flow). (a) Average routing state. (b) Number of routing table entries.

TABLE I

MAXIMUM ROUTING STATE OF S4 AND BVR

\begin{tabular}{|l|l|l|l|}
\hline & $\max$ S4 state (B) & $\max$ BVR state (B) & $\max$ S4 routing entries \\
\hline HD & 680 & 960 & 136 \\
\hline LD & 715 & 920 & 143 \\
\hline
\end{tabular}

We first compare the total amount of routing state in bytes between S4 and BVR.

Fig. 13(a) shows the average routing state over all nodes. We make the following observations. First, network density has little impact on the routing state of $\mathrm{S} 4$, but has large impact on BVR. This is because in S4 the local cluster sizes are not sensitive to network density (when density increases, the scope tends to decrease), while in BVR each node stores the coordinates of its neighbors and its routing state increases with density. Second, the amount of routing state in BVR increases with the number of beacons. In comparison, S4's routing state does not necessarily increase with the number of beacons since increasing the number of beacons reduces the local cluster size. Third, when the number of beacons is $32(\approx \sqrt{1000})$ or above, the routing state in S4 is less than BVR. Similar results have been observed in other TOSSIM configurations as well as MATLAB simulation results in Section IV.

Fig. 13(b) further shows the number of entries in the beacon routing table, local cluster table, and neighbor coordinate table. The beacon table curves of S4 and BVR overlap since it is common for both. Note that although the coordinate tables in BVR have fewer entries than the cluster tables in S4, the total size of the coordinate tables are generally larger since the size of each coordinate table entry is proportional to the number of beacons.

Table I shows maximum routing state of S4 and BVR under high density and low density. The maximum number of routing entries is around 4.5 times of $\sqrt{1000}$ (the expected average cluster size), but still an order of magnitude smaller than 1000 (the flat routing table size) in shortest path routing. This suggests that random beacon selection does a reasonably good job in limiting worst-case storage cost.

Node load: Fig. 14 shows the average number of packets that each node transmits, under lossless links and 5-flow traffic. Fig. 14(a) shows the beacon node load, and Fig. 14(b) shows nonbeacon node load. We observe that in S4 both beacon nodes and nonbeacon nodes experience lower load than those nodes in BVR. This is due to lower routing stretch and transmission

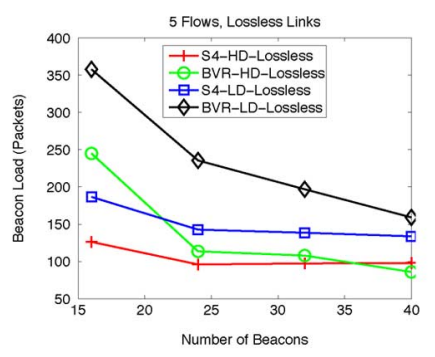

(a)

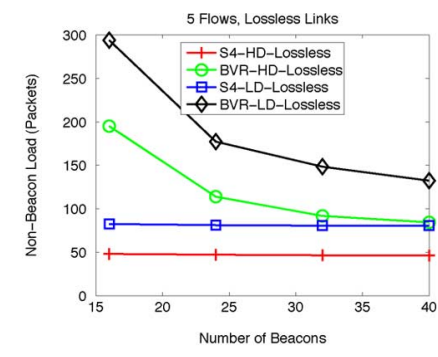

(b)
Fig. 14. Node load of data traffic under different numbers of beacons and network densities with lossless Links (five flows). (a) Beacon load. (b) Nonbeacon load.

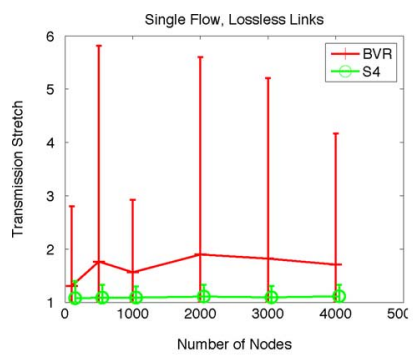

(a)

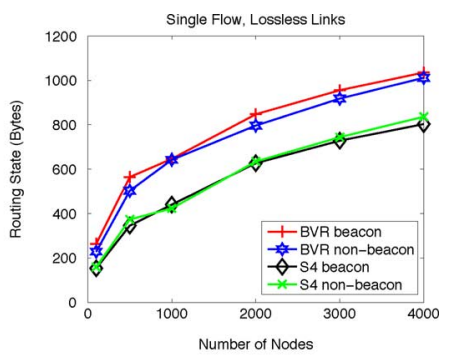

(b)
Fig. 15. Comparison under different network sizes. (a) Transmission stretch. (b) Routing state.

stretch in S4. In addition, we observe that in S4, the beacon load is within a factor of 1.5-2 of nonbeacon load, which means the load is reasonably balanced among beacon and nonbeacon nodes. Similar results are observed under single-flow traffic.

2) Varying Network Size: We also evaluate the performance and scalability of S4 when the network size changes from 100 to 4000 . In both $\mathrm{S} 4$ and BVR, for a network of $N$ nodes, we select $K \approx \sqrt{N}$ nodes as beacon nodes for fair comparison. In the interest of space, we only present results under lossless links and a single flow.

Fig. 15(a) shows the average transmission stretch of S4 and BVR under different network sizes. The error bars represent fifth and 95th percentiles. S4 achieves smaller transmission stretches and smaller variations in the stretches. In BVR, packets experience higher medium stretch and higher stretch variation due to greedy forwarding and scoped flooding.

Fig. 15(b) shows the average routing state. For both $\mathrm{S} 4$ and BVR, the routing state tends to increase with $O(\sqrt{N})$. This suggests both S4 and BVR are scalable with network sizes. In particular, even when the network size is 4000 , the majority of nodes can store the routing state in a small portion of a 4-kB RAM (the RAM size on Mica2 motes we experimented with). Moreover, S4 uses less routing state than BVR when the number of beacon nodes is $\sqrt{N}$ because the coordinate table size in BVR is linear to the number of beacon nodes.

To further study the performance of S4 in smaller networks, we compare S4 and BVR in networks of 100 nodes. Due to space limitation, we only include the results for the case of single flow traffic with lossless links. Table II shows that in 100-node networks, S4 outperforms BVR in terms of routing success rate, routing stretch, transmission stretch, and routing state. S4 incurs more control overhead than BVR due to the extra SDV traffic, 


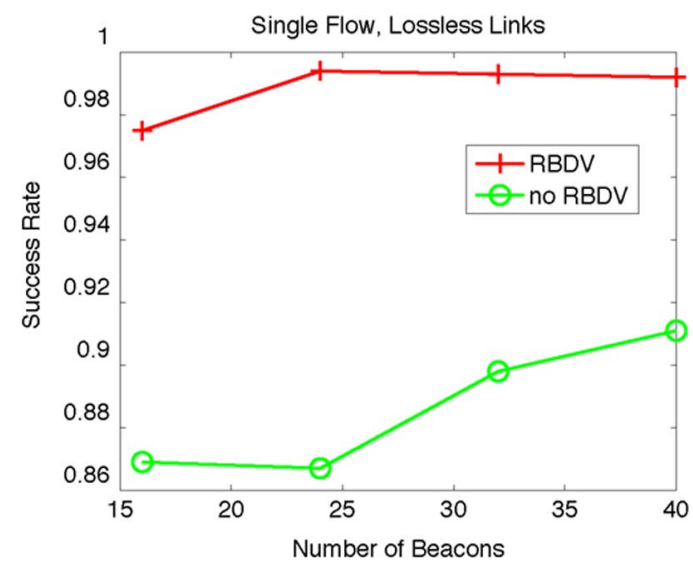

Fig. 16. Impact of RBDV on success rate (1000 nodes, low density).

TABLE II

PERFORMANCE COMPARISON IN 100-NODE NETWORKS

\begin{tabular}{|c|l|l|l|l|l|}
\hline & $\begin{array}{l}\text { success } \\
\text { rate }\end{array}$ & $\begin{array}{l}\text { routing } \\
\text { stretch }\end{array}$ & $\begin{array}{l}\text { transmission } \\
\text { stretch }\end{array}$ & $\begin{array}{l}\text { control } \\
\text { traffic (B) }\end{array}$ & $\begin{array}{l}\text { routing } \\
\text { state (B) }\end{array}$ \\
\hline S4 & 1 & 1.07 & 1.08 & 96 & 158 \\
\hline BVR & 0.994 & 1.20 & 1.31 & 46 & 232 \\
\hline
\end{tabular}

though its overall control traffic (after including location directory setup traffic) is still comparable to that of BVR.

\section{B. Impact of $R B D V$}

Next, we evaluate resilient beacon distance vector (RBDV). Again, we use 1000-node networks. We turn off periodic transmissions of beacon and SDV messages so that the failed transmissions of these messages have to be recovered using RBDV, but not using periodic beacon transmissions. This is an interesting scenario to consider because we want to minimize the frequency of periodic broadcasts while still achieving high delivery rate. Each beacon broadcasts once. Other nodes who receive a beacon packet further broadcast it. Similarly, a nonbeacon node broadcasts its own scoped distance vector once. A node further broadcasts a SDV only if it is inside the scope.

We simulate for single-flow data traffic with lossless links and compare the routing success rate between the case with and without RBDV. In both cases, DLF is enabled. Packet collisions are common when nodes broadcast beacon packets or scoped distance vectors. As shown in Fig. 16, without RBDV, the success rate is around $90 \%$. With RBDV, the success rate is improved to close to $100 \%$ because RBDV helps to improve accuracy of the routing tables.

\section{Impact of Node Failures}

We now evaluate the performance of S4 under node failures. In our evaluation, we first establish routes using SDV and RBDV as usual. Then, we randomly kill a certain number of nodes after route initialization is completed and evaluate the success rate of routing data traffic. Since BVR does not incrementally update routing state between rounds, to make fair comparison, we disable incremental routing update in S4, and completely rely on DLF to recover failures. The performance of S4 would be even better if we incrementally update routing states upon failures. We distinguish between beacon and nonbeacon failures

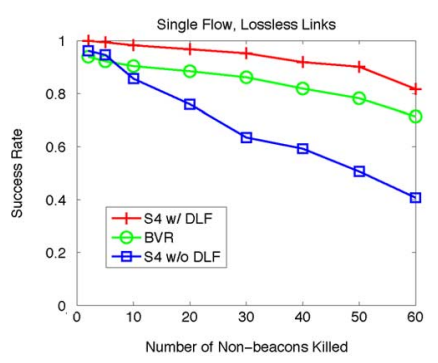

(a)

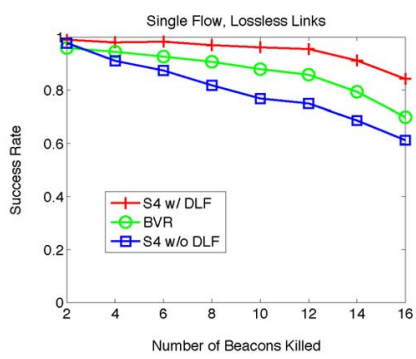

(b)
Fig. 17. Impact of DLF on success rate (1000 nodes, 32 beacons, low density) (a) Random nonbeacon failures. (b) Random beacon failures.

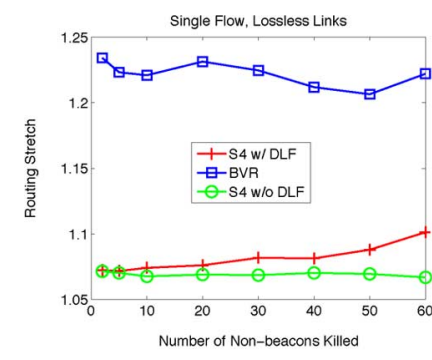

(a)

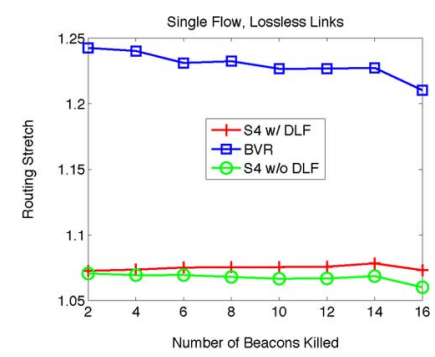

(b)
Fig. 18. Impact of DLF on routing stretch (1000 nodes, 32 beacons, low density). (a) Random nonbeacon failures. (b) Random beacon failures.

and show the results under lossless links and single-flow traffic in comparison to BVR. By default, scoped flooding is enabled in BVR.

Fig. 17 shows that failure recovery can significantly increase the success rate under both nonbeacon and beacon failures. DLF in S4 is more effective than the scoped flooding in BVR for the following reasons. First, scoped flooding results in packet collisions. Second, S4 uses unicast for data transmissions and benefits from link-layer retransmissions. Third, if some node between the beacon and destination fails, DLF can recover such failures, while scoped flooding cannot.

Next, we compute the average routing stretch over all successfully delivered packets. As we expect, packets going through failure recovery take longer than normal paths. Interestingly, as shown in Fig. 18, the average routing stretch is only slightly higher than the case of no failure recovery, which indicates the robustness of S4.

\section{Summary}

Our TOSSIM evaluation further confirms that $\mathrm{S} 4$ is scalable to large networks: The average routing state scales with $O(\sqrt{N})$ in an $N$-node network. The average routing and transmission stretches in S4 are around 1.1-1.2. This is true not only in lossless networks under single flow traffic, but also under lossy wireless medium, packet collisions arising from multiple flows, and significant failures. This demonstrates that $\mathrm{S} 4$ is efficient and resilient. In comparison, the performance of BVR is sensitive to wireless channel condition. Even under loss-free networks, it may not provide $100 \%$ delivery guarantee due to possible packet collisions incurred in scoped flooding. Its routing and transmission stretches also increase with wireless losses and failures. 


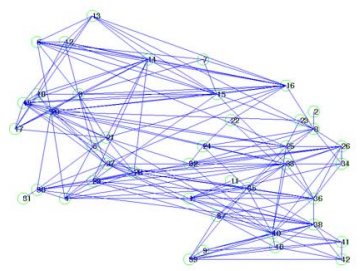

(a)

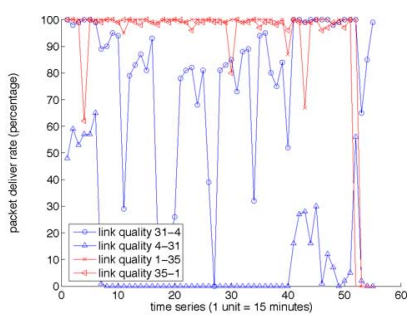

(b)
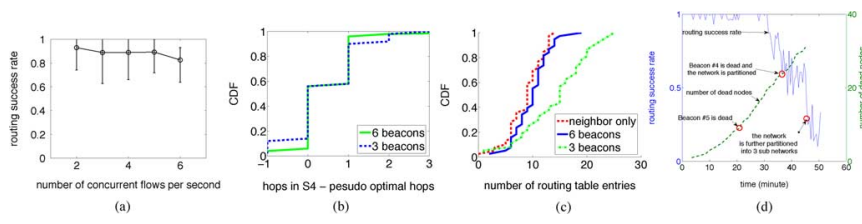

Fig. 20. Experiments on the 42-node testbed. (a) Routing success rate under multiple concurrent flows. (b) CDF of the hop count difference to pseudo optimal. (c) Routing table size. (d) Routing performance under node failure.

TABLE III

Routing Success RATE IN THE 42-NoDE TESTBED

\section{Testbed Evaluation}

To demonstrate the feasibility of S4 in real wireless networks, we deploy the S4 prototype on a testbed of 42 mica2 motes with $915-\mathrm{MHz}$ radios on the fifth floor of the ACES building at the University of Texas at Austin. While the testbed is only a moderate size and cannot stress-test the scalability of S4, it does allow us to evaluate $\mathrm{S} 4$ under realistic radio characteristics and failures. We adjust the transmission power to $-17 \mathrm{dBm}$ for all control and data traffic to obtain an interesting multihop topology. With such a power level, the testbed has a network diameter of around 4 to 6 hops, depending on the wireless link quality. Eleven motes are connected to the MIB600 Ethernet boards that we use for logging information. They also serve as gateway nodes to forward commands and responses for the remaining 31 battery-powered motes ${ }^{3}$.

Fig. 19(a) shows a snapshot of the network topology. We measure packet delivery rates by sending broadcast packets on each mote one by one. Two motes have a link if the delivery rates on both directions are above $30 \%$. Because no two nodes will broadcast packets at the same time, the measurement result is optimistic in the sense that channel contention and network congestion is not considered. The average node degree is 8.7. We observe that a short geographic distance between two motes does not necessarily lead to good link quality. Some of the links are very asymmetric, and their qualities vary dramatically over time. As shown in Fig. 19(b), some of the links are highly asymmetric and their qualities vary dramatically over time. For example, the link qualities between motes 4 and 31 fluctuate as time goes by and are quite asymmetric, while link qualities between motes 1 and 15 are fairly stable to $100 \%$ delivery rate, until in the last $1 \mathrm{~h}$ when they suddenly drop to almost $0 \%$. Such link characteristics allow us to stress-test the performance and resilience of S4.

\section{A. Routing Performance}

We randomly preselect six nodes out of 42 nodes as beacon nodes for S4. The distance from any node to its closest beacon is at most two hops. After 10 min of booting up all the motes, we randomly select source and destination pairs to evaluate routing performance. The sources are selected from all 42 motes, and the destinations are selected from the 11 motes that are connected to the Ethernet boards. All destinations dump the packet

\footnotetext{
${ }^{3}$ Unfortunately, we are unable to compare S4 against BVR in our testbed. Current BVR implementation requires all motes have Ethernet boards connected to send and receive routing commands. However, our testbed only has 11 motes with Ethernet connections, which would make the evaluation less interesting.
}

\begin{tabular}{|c|c|c|}
\hline time period & \# pkts per sec & routing success rate \\
\hline $0-70.1 \mathrm{~min}$ & 1 & $99.9 \%$ \\
$70.1-130.2 \mathrm{~min}$ & 2 & $99.1 \%$ \\
\hline
\end{tabular}

delivery confirmation through UART to the PC for further analysis. For each routing request, unless the source is connected to an Ethernet board, we choose the gateway mote that is the closest to the source to forward a command packet. The command packet is sent with the maximum power level and up to five retransmissions so that the source is very likely to receive it. Upon receiving the routing request, the source will send back a response packet with the maximum power level and potential retransmissions to acknowledge successful reception of the routing request. Each routing request is tagged with a unique sequence number to make the operation idempotent. After the command traffic, the data packet will be sent at a lower power level in order to have an interesting multihop network topology.

We send routing requests at one packet per second for the first 70 min (altogether 4210 packets), and then double the sending rate thereafter for another $60 \mathrm{~min}$ (altogether 7701 packets). As shown in Table III, the routing success rate is $99.1 \%-99.9 \%$ and consistent over time. This demonstrates the resilience of S4 in a real testbed.

Next, we use multiple constant bit rate (CBR) flows to increase the network load. In each multiple flow test, we randomly pick $n$ source destination pairs, and instrument the sources to send consecutive packets at the rate of one packet per $s$ s. This is essentially having $n / s$ random flows per second. The flows start after a predefined idle period to avoid potential collisions with the command traffic. We choose $s=2$ and test up to six concurrent flows (i.e., $n$ is up to 12). For each experiment, we repeat it for 10 times. Fig. 20(a) plots the median routing success rates in different flow settings. The error bars indicate the best-case and worst-case routing success rate. We see the median success rate gracefully degrades with an increasing number of concurrent flows. Our log collected from the gateway motes indicates that some of the failures are due to the limitation of a single forwarding buffer per node. Such failure happens when two or more flows try to concurrently route through the same node. Note that this is not a protocol limitation in S4. We could remove many such failures by having a more complete implementation that supports multiple forwarding buffers.

Finally, we study the routing efficiency of S4. Note that it is impossible to calculate the true routing stretch in a real wireless network because the topology is always changing, and the packet loss rates depend on the traffic pattern so that the optimal routes are changing, too. Instead, we compare S4 
against the pseudo-optimal hop count metric. The pseudo-optimal hop count of a route is defined as the shortest path length in a snapshot of the network topology. In our experiment, we use broadcast-based active measurement to obtain the pair-wise packet delivery rates before the routing test starts. The delivery rates are averaged over a $1-\mathrm{h}$ measurement period. Note that the real optimal routes could be either better or worse than the pseudo-optimal ones due to topology changes, and the delivery rates tend to be optimistic due to no packet collision in the measurement. The routing tests follow the measurement within $30 \mathrm{~min}$. We randomly select source and destination pairs and send routing requests at one packet per second for $5000 \mathrm{~s}$. Then, we change the number of beacons from 6 to 3 and repeat the same test. The shortest paths from the topology snapshot are computed offline. Fig. 20(b) shows that more than $95 \%$ of the routes are within one-hop difference from the pseudo-optimal hops under six beacons. Interestingly, S4 sometimes achieves better performance than the pseudo-optimal scheme. This is because during the 5000-s routing experiment, $\mathrm{S} 4$ adapts to the change of topology so that it can take advantage of new links and reduce path lengths. The number of beacons also has both positive and negative effects on routing performance. When fewer beacons are selected, the nodes tend to have larger routing tables so that more nodes can be reached via the shortest paths; however, having fewer beacons also leads to more control traffic so that the link estimator will have a more pessimistic estimation on link quality due to packet collision. Underestimating link quality apparently hurts the routing performance.

In the same experiment, we also study the routing state per node in S4. Fig. 20(c) compares the numbers of local routing table entries used under six and three beacons. Using six beacons yields smaller routing tables. A node in $\mathrm{S} 4$ has local routing state toward its neighbor unless the neighbor is a beacon node. Therefore, the number of routing entries at each node is generally larger than the number of its neighbors. We find that on average, when six beacons are used, the routing table has only three more entries than a typical neighborhood table, which suggests that the routing state in S4 is small.

\section{B. Routing Under Node Failures}

To stress-test the resilience of S4, we artificially introduce node failure in our testbed. We randomly select nongateway motes to kill one by one and study the routing performance. We send one routing request per second for $50 \mathrm{~min}$, altogether generating 3000 packets. The source node is randomly selected from the current live nodes, and the destination is one of the gateway motes. Note that we do not start any SDV update or beacon broadcast after the initial setup stage in order to study the effectiveness of the failure recovery mechanism alone. As shown in Fig. 20(d), in the first 30 min, even when 20 motes are killed, including a beacon node, the routing success rate is still close to $100 \%$. The routing success rate starts to drop after 30 min due to congestion at some bottleneck links. When the second beacon is killed, the network is partitioned and more routing failures are expected. The third major performance degradation occurs after all 31 nongateway motes are dead, which causes further network partitions. These results show that $\mathrm{S} 4$ is resilient to failures.

\section{Summary}

Our evaluation in the 42-node testbed shows that S4 achieves close to $100 \%$ routing success rate in a normal condition with a single flow. Meanwhile, S4 degrades gracefully with an increasing number of packet collisions (in multiple concurrent flows) and node failures.

\section{CONCLUSION}

We present $\mathrm{S} 4$ as a scalable routing protocol in large wireless networks to simultaneously minimize routing state and routing stretch in both normal conditions and under node or link failures. S4 incorporates a scoped distance vector protocol (SDV) for intracluster routing, a resilient beacon distance vector protocol (RBDV) for intercluster routing, and distance-guided local failure recovery (DLF) for achieving resilience under failures and topology changes. S4 uses small amounts of routing state to achieve a worst-case routing stretch of 3 and an average routing stretch of close to 1. Evaluation across a wide range of scenarios, using high-level and packet-level simulators, and real testbed deployment show that S4 achieves scalability, efficiency, and resilience.

\section{ACKNOWLEDGMENT}

The authors thank D. Ganesan, R. Mahajan, and the anonymous reviewers for their helpful comments on the earlier versions of this paper. They thank R. Fonseca and S. Ratnasamy for sharing their BVR source code, and thank Y. Zhang for the helpful discussion.

\section{REFERENCES}

[1] D. Aguayo, J. Bicket, S. Biswas, G. Judd, and R. Morris, "Link-level measurements from an 802.11 b mesh network," in Proc. ACM SIGCOMM, Aug. 2004, pp. 121-132.

[2] P. Bose, P. Morin, I. Stojmenovic, and J. Urrutia, "Routing with guaranteed delivery in ad hoc wireless networks," in Proc. 3rd ACM DIALM, Aug. 1999, pp. 48-55.

[3] M. Caesar, M. Castro, E. B. Nightingale, G. O'Shea, and A. Rowstron, "Virtual ring routing: Network routing inspired by DHTs," in Proc. ACM SIGCOMM, Sep. 2006, pp. 351-362.

[4] L. Cowen, "Compact routing with minimum stretch," J. Algor., vol. 38, no. 1, pp. 170-183, Jan. 2001.

[5] R. Fonseca, S. Ratnasamy, J. Zhao, C. T. Ee, D. Culler, S. Shenker, and I. Stoica, "Beacon vector routing: Scalable point-to-point routing in wireless sensornets," in Proc. NSDI, May 2005, pp. 329-342.

[6] H. Frey and I. Stojmenovic, "On delivery guarantees of face and combined greedy-face routing in ad hoc and sensor networks," in Proc. ACM MobiCom, Sept. 2006, pp. 390-401.

[7] K. Gabriel and R. Sokal, "A new statistical approach to geographic variation analysis," Syst. Zoology, pp. 259-278, 1969.

[8] M. Gerla, X. Hong, and G. Pei, "Landmark routing for large ad hoc wireless networks," in Proc. IEEE Globecom, Nov. 2000, vol. 3, pp. 1702-1706.

[9] Z. J. Haas, M. R. Pearlman, and P. Samar, "The zone routing protocol (ZRP) for ad hoc networks," IETF MANET Working Group, InternetDraft, Jul. 2002.

[10] J. Hill, R. Szewczyk, A. Woo, S. Hollar, D. Culler, and K. Pister, "System architecture directions for networked sensors," in Proc. ASPLOS, 2000, pp. 93-104.

[11] D. Johnson, D. Maltz, and J. Broch, "DSR: The dynamic source routing protocol for multihop wireless ad hoc networks," in Ad Hoc Networking. Boston, MA: Addison-Wesley, 2001.

[12] B. Karp and H. Kung, "GPSR: Greedy perimeter stateless routing for wireless networks," in Proc. ACM MobiCom, Aug. 2000, pp. 243-254.

[13] Y.-J. Kim, R. Govindan, B. Karp, and S. Shenker, "Geographic routing made practical," in Proc. NSDI, May 2005, pp. 217-230. 
[14] Y.-J. Kim, R. Govindan, B. Karp, and S. Shenker, "On the pitfalls of geographic face routing," in Proc. 3rd ACM/SIGMOBILE DIALMPOMC, 2005, pp. 34-43.

[15] F. Kuhn, R. Wattenhofer, Y. Zhang, and A. Zollinger, "Geometric ad-hoc routing: Of theory and practice," in Proc. ACM PODC, 2003, pp. 63-72.

[16] F. Kuhn, R. Wattenhofer, and A. Zollinger, "Asymptotically optimal geometric mobile ad-hoc routing," in Proc. ACM DIALM, 2002, pp. 24-33.

[17] B. Leong, B. Liskov, and R. Morris, "Geographic routing without planarization," in Proc. NSDI, May 2006, p. 25.

[18] B. Leong, S. Mitra, and B. Liskov, "Path vector face routing: Geographic routing with local face information," in Proc. IEEE ICNP, Nov. 2005.

[19] P. Levis, N. Lee, M. Welsh, and D. Culler, "TOSSIM: Accurate and scalable simulation of entire TinyOS applications," in Proc. ACM SenSys, 2003, pp. 126-137.

[20] C. Newport, D. Kotz, Y. Yuan, R. S. Gray, J. Liu, and C. Elliott, "Experimental evaluation of wireless simulation assumptions," Simulation, vol. 83, no. 9, pp. 643-661, Sep. 2007.

[21] J. Newsome and D. Song, "GEM: Graph embedding for routing and data-centric storage in sensor networks without geographic information," in Proc. ACM SenSys, Nov. 2003, pp. 76-88.

[22] C. E. Perkins and P. Bhagwat, "Highly dynamic destination-sequenced distance-vector routing (DSDV) for mobile computers," in Proc. ACM SIGCOMM, 1994, pp. 234-244.

[23] C. E. Perkins and E. M. Royer, "Ad hoc on-demand distance vector routing," in Proc. 2nd IEEE Workshop Mobile Comput. Syst. Appl., Feb. 1999, pp. 90-100.

[24] A. Post and D. Johnson, "Self-organizing hierarchical routing for scalable ad hoc networking," Rice CS Tech. Rep. TR04-433, 2004.

[25] A. Rao, S. Ratnasamy, C. Papadimitriou, S. Shenker, and I. Stoica, "Geographic routing without location information," in Proc. ACM MobiCom, Sep. 2003, pp. 96-108.

[26] M. Thorup and U. Zwick, "Approximate distance oracles," in Proc. ACM STOC, 2001, pp. 183-192.

[27] M. Thorup and U. Zwick, "Compact routing schemes," in Proc. SPAA, Jul. 2001, pp. 1-10.

[28] G. Toussaint, "The relative neighborhood graph of a finite planar set," Pattern Recognition, pp. 261-268, 1980.

[29] P. F. Tsuchiya, "The landmark hierarchy: A new hierarchy for routing in very large networks," in Proc. ACM SIGCOMM, 1988, pp. 35-42.

[30] A. Woo, T. Tong, and D. Culler, "Taming the underlying challenges of reliable multhop routing in sensor networks," in Proc. ACM SenSys, Nov. 2003, pp. 14-27.

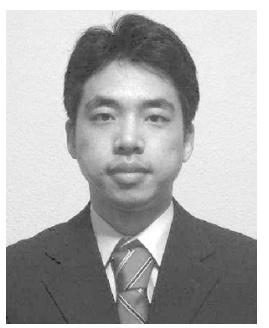

Feng Wang received the Bachelor's degree in computer science from the University of Science and Technology of China, Hefei, China, in 1997, and the Master's and Ph.D. degrees in computer science from the University of Texas at Austin in 2002 and 2007, respectively.

He is currently a Member of Technical Staff in Meraki, Inc., San Francisco, CA. He is part of a team responsible for designing and implementing firmware running on all Meraki access points. He designed and implemented an algorithm that runs on Meraki's cloud-based controllers to optimize channel assignment for wireless networks. His research interests are in wireless networks, particularly mesh/ad hoc and sensor networks. His thesis topic is on wireless mesh network management.

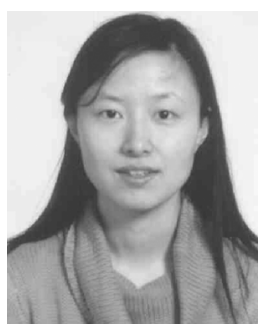

Lili Qiu (SM'07) received the Ph.D. degree in computer science from Cornell University, Ithaca, NY, in 2001.

She is an Assistant Professor with the Department of Computer Sciences, University of Texas at Austin. She was a Researcher at Microsoft Research, Seattle, WA, from 2001 to 2004. Her research interests are in Internet and wireless networking, with special focuses on Internet measurement, overlay routing, content distribution, and wireless network performance and management.

Dr. Qiu is a recipient of the NSF CAREER Award in 2006.

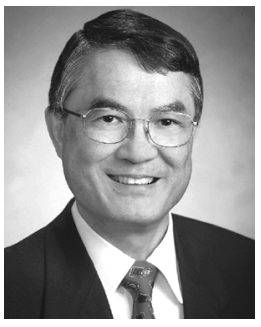

Simon Lam (F'85) received the B.S.E.E. degree with Distinction from Washington State University, Pullman, in 1969, and the M.S. and Ph.D. degrees in engineering from the University of California, Los Angeles, in 1970 and 1974, respectively.

From 1974 to 1977, he was a Research Staff Member with the IBM T. J. Watson Research Center, Yorktown Heights, NY. Since 1977, he has been on the faculty of the University of Texas at Austin, where he is Professor and Regents Chair in computer science.

Prof. Lam is has been a Fellow of the Association for Computing Machinery (ACM) since 1998 and a Member of the National Academy of Engineering since 2007. He received the 2004 SIGCOMM Award for lifetime contribution to the field of communication networks, the 2004 ACM Software System Award for inventing secure sockets and prototyping the first secure sockets layer (named Secure Network Programming), the 2004 W. Wallace McDowell Award from the IEEE Computer Society, as well as the 1975 Leonard G. Abraham Prize and the 2001 William R. Bennett Prize from the IEEE Communications Society.

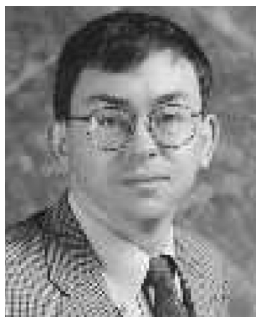

Jonathan M. Smith (F'01) received the Ph.D. degree in computer science from Columbia University, New York, NY, in 1989

$\mathrm{He}$ is the Olga and Alberico Pompa Professor of Engineering and Applied Science at the University of Pennsylvania, Philadelphia. He was a Program Manager at the Defense Advanced Research Projects Agency (DARPA), Arlington, VA, where he managed programs in areas ranging from network protocols and network security to quantum information science. His current research interests are programmable network infrastructures and network security.

Prof. Smith served on the PCAST Network and Information Technology Technical Advisory Group from 2006 to 2009 and is currently a member of the NRC Board on Army Science and Technology. 